\title{
Nationwide Implementation of Robotic Minimally Invasive Surgery for Endometrial Cancer Increases Survival and Reduces Complications
}

Jørgensen S.L., Mogensen O. , Wu CS., Korsholm M., lachina M., Lund K., Jensen P.T.

Affiliations: Odense University Hospital, Denmark; University of Southern Denmark, Denmark; Karolinska Institute, Sweden

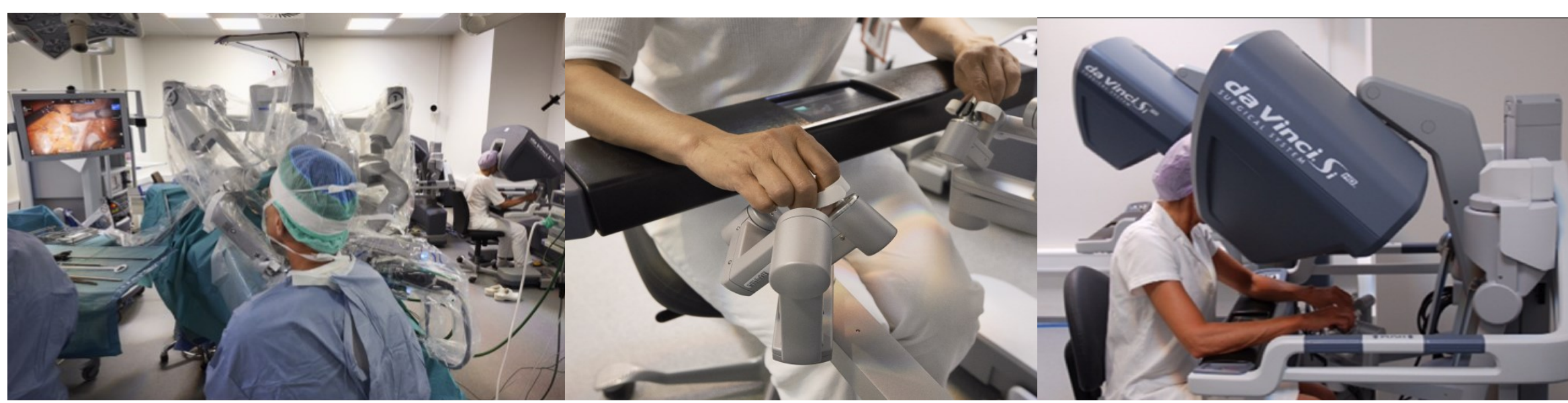




\section{Aim \& Design}

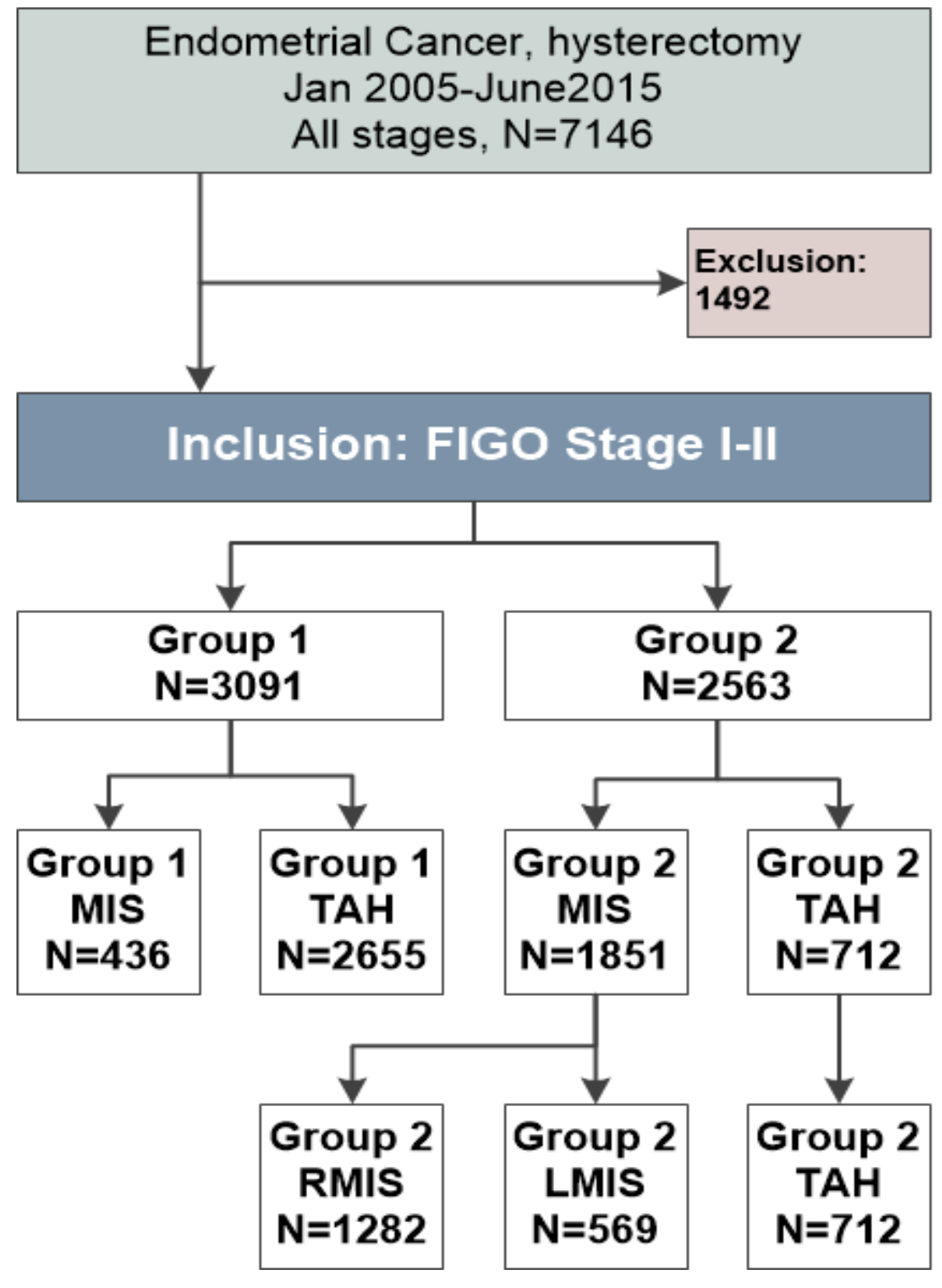

To determine if a nationwide implementation of robotic minimally invasive surgery influenced the risk of severe complications and survival among women with early-stage endometrial cancer

The individual woman was allocated to Group 1 if operated BEFORE and to

Group 2 if operated AFTER RMIS implementation in her county 


\section{Nationwide implemetation of RMIS} transitioned the surgical approach

\section{From $97 \%$ open to $95 \%$ MIS over a decade}

MIS reduces severe complications

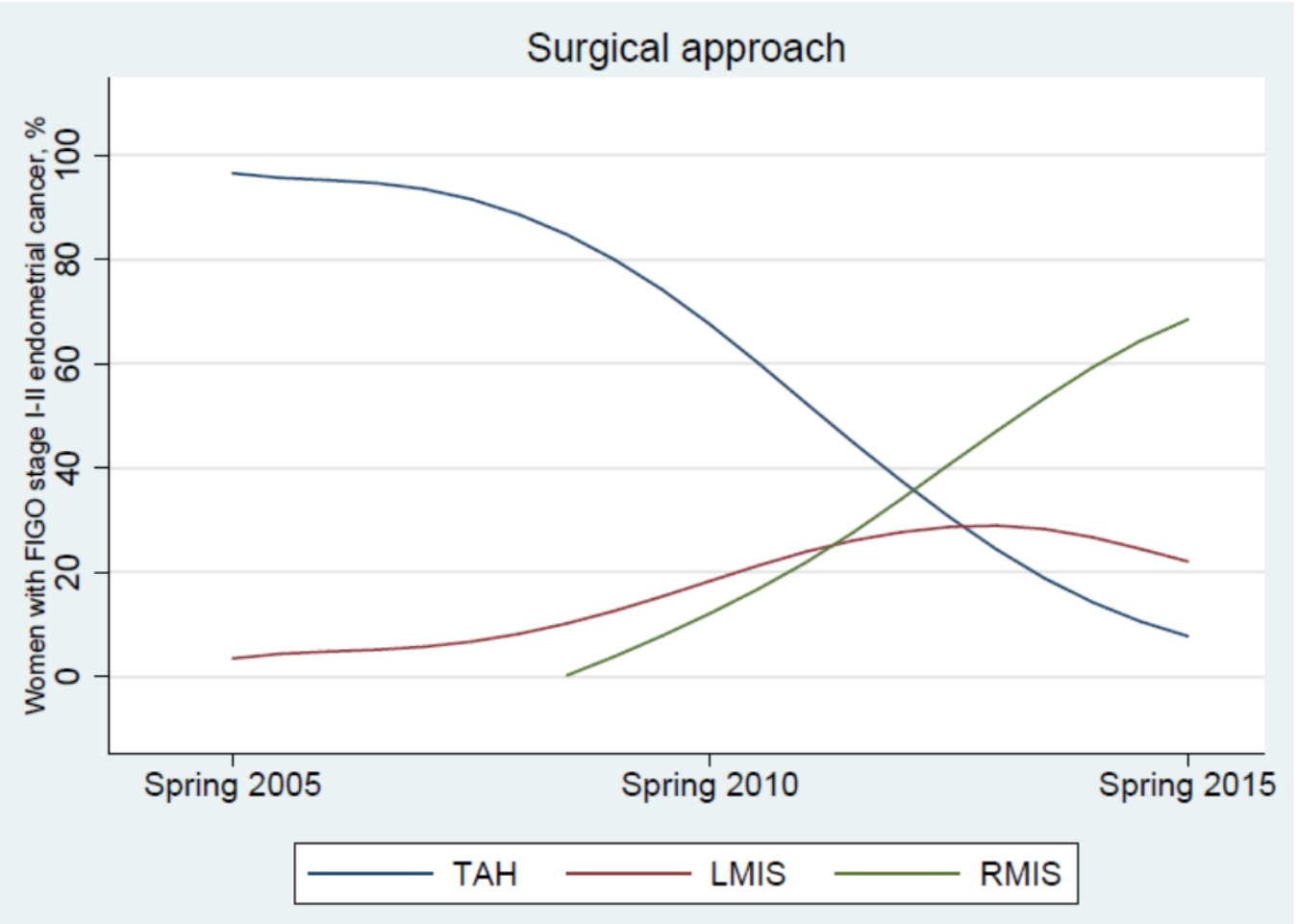

\begin{tabular}{|c|c|c|c|}
\hline & $\begin{array}{c}\text { Severe } \\
\text { complications }\end{array}$ & Multivariate log & jistic regression \\
\hline Group 1, overall & $7.3 \%$ & OR, 1.39 & $(95 \%$ Cl 1.1-1.74) \\
\hline Group 2, overall & $6.2 \%$ & \multicolumn{2}{|c|}{ Reterence } \\
\hline Group 2, TAH & $11.4 \%$ & OR, 2.91 & $(95 \% \mathrm{Cl} 2.01-4.23)$ \\
\hline Group 2, LMIS & $5.1 \%$ & OR, 1.39 & $(95 \% \mathrm{Cl} 0.87-2.23)$ \\
\hline Group 2, RMIS & $3.9 \%$ & \multicolumn{2}{|c|}{ Reference } \\
\hline
\end{tabular}

TAH, Total Abdominal Hysterectomy

LMIS, Laparoscopic Minimally Invasive Surgery

RMIS, Robotic Minimally Invasive Surgery

Group 1, Before RMIS implementation

Group 2, After RMIS implementation 


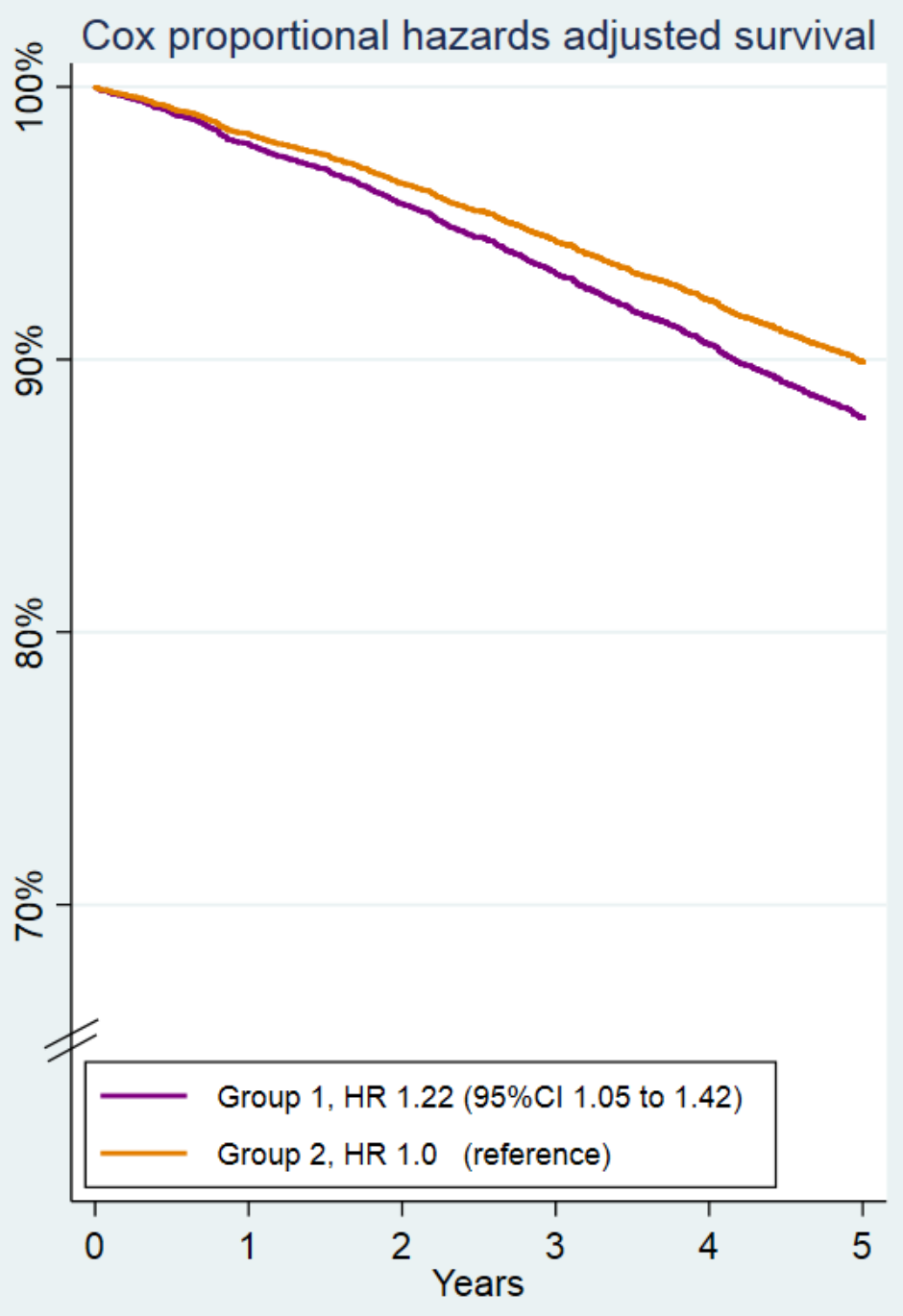

The 5-year survival was significantly lower before RMIS was implemented

The 5-year survival was significantly lower following TAH

No difference was found between LMIS and RMIS

TAH, Total Abdominal Hysterectomy

LMIS, Laparoscopic Minimally Invasive Surgery

RMIS, Robotic Minimally Invasive Surgery

Group 1, Before RMIS implementation

Group 2, After RMIS implementation

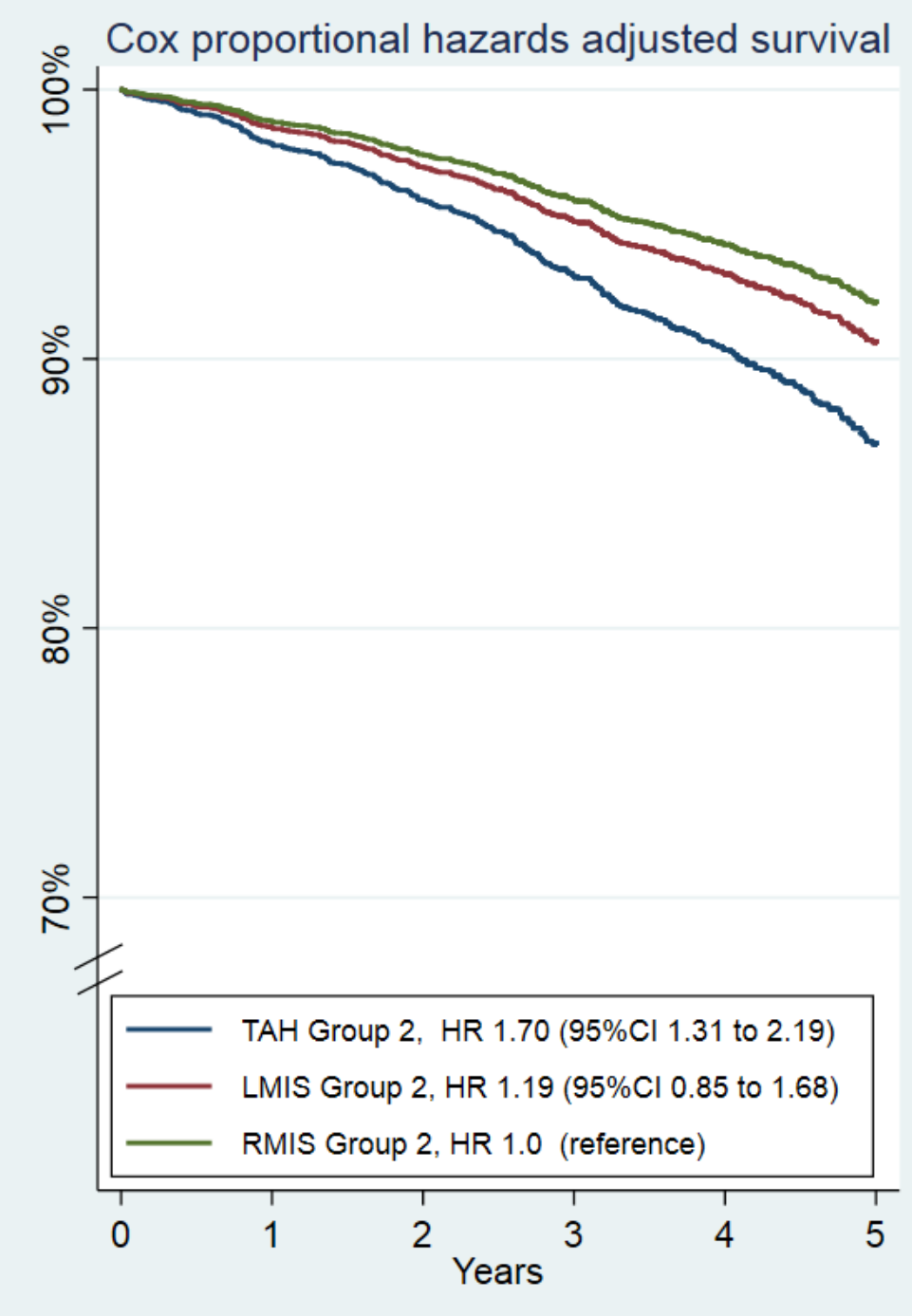

University of Southern Denmark 


\section{Conclusion}

The Danish nationwide implementation of robotic surgical availability enabled a paradigm shift towards minimally invasive surgery

This translated into reduced risk of severe complications and increased survival.

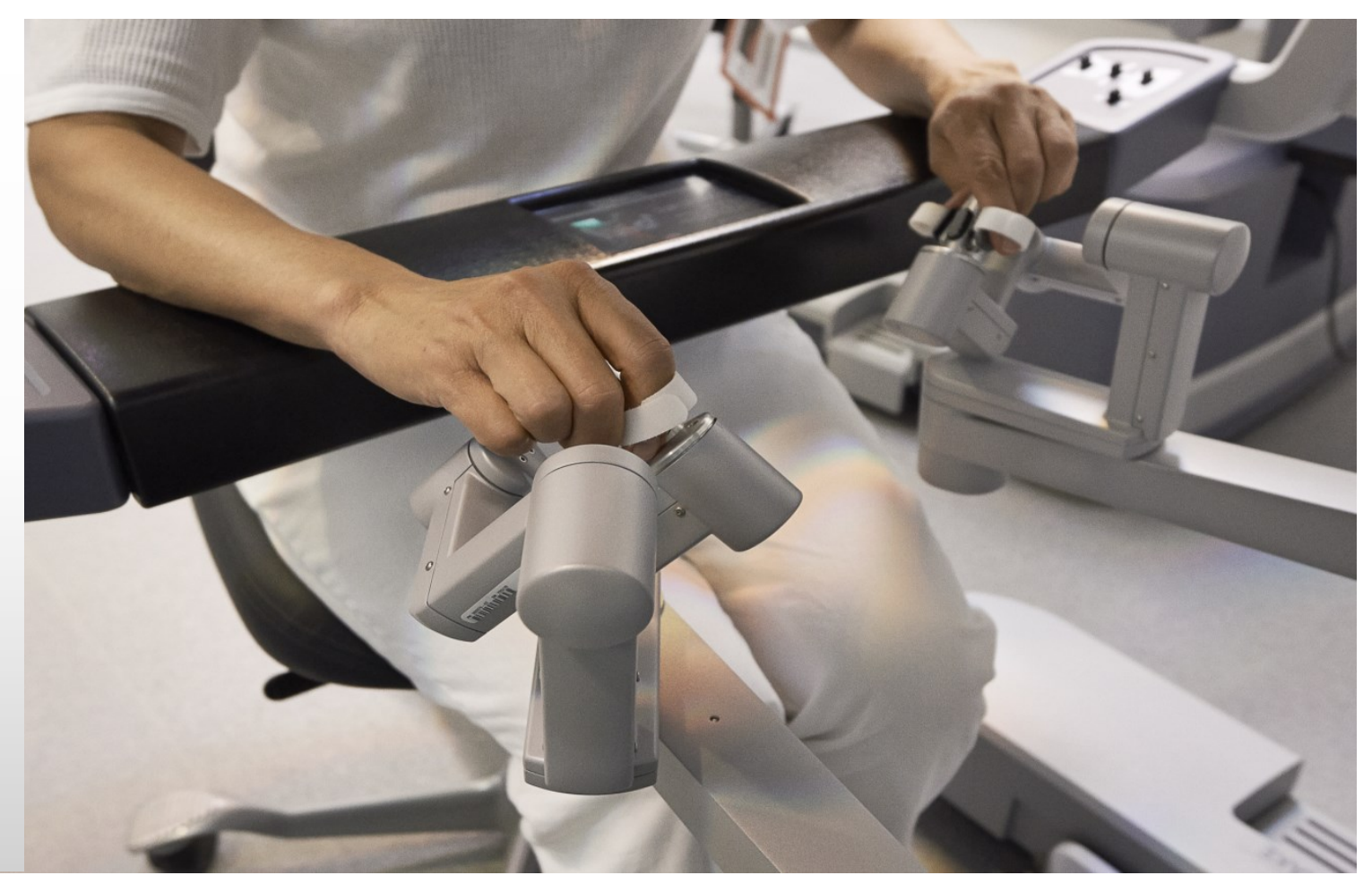

